\title{
KRAS and BRAF mutations are rare and related to DNA mismatch repair deficiency in gastric cancer from the East and the West: Results from a large international multicentre study
}

N C T van Grieken ${ }^{1,15}$, T Aoyma ${ }^{2,15}$, P A Chambers ${ }^{3}$, D Bottomley ${ }^{4}$, L C Ward ${ }^{4}$, I Inam ${ }^{4}$, T E Buffart ${ }^{1}$, K Das $^{5}$, T Lim ${ }^{6}$, B Pang ${ }^{7}$, S L Zhang ${ }^{5}$, I B Tan ${ }^{8}$, B Carvalho ${ }^{1}$, D A M Heideman ${ }^{1}$, Y Miyagi ${ }^{9}$, Y Kameda ${ }^{10}$, T Arai $^{11}$, G A Meijer ${ }^{1}$, A Tsuburaya ${ }^{2}$, P Tan ${ }^{5,12,13,14}$, T Yoshikawa*,2 and H I Grabsch ${ }^{*} 4$

\begin{abstract}
${ }^{1}$ Department of Pathology, VU University Medical Center, Po Box 7057, 1007 MB Amsterdam, The Netherlands; ${ }^{2}$ Department of Gastrointestinal Surgery, Kanagawa Cancer Center, 1-1-2 Nakao, Asahi-ku, Yokohama 241-8515, Japan; ${ }^{3}$ Cancer Research UK Centre Genomics Facility, Leeds Institute of Molecular Medicine, University of Leeds, St James's University Hospital, Beckett Street, Leeds LS9 7TF, UK; ${ }^{4}$ Section of Pathology and Tumour Biology, Leeds Institute of Molecular Medicine, University of Leeds, St James's University Hospital, Beckett Street, Leeds LS9 7TF, UK; ${ }^{5}$ Cancer and Stem Cell Biology, Duke-NUS Graduate Medical School, 8 College Road, Singapore 169857, Singapore; ${ }^{6}$ Department of Pathology, National Cancer Centre Singapore, 11 Hospital Drive, Singapore 169610, Singapore; ${ }^{7}$ Department of Pathology, National University Hospital, 5 Lower Kent Ridge Road, Singapore 119074, Singapore; ${ }^{8}$ Department of Medical Oncology, National Cancer Centre Singapore, 11 Hospital Drive, Singapore 169610, Singapore; ${ }^{9}$ Molecular Pathology and Genetics Division, Kanagawa Cancer Center Research Institute, 1-1-2 Nakao, Asahi-ku, Yokohama 241-8515, Japan; ${ }^{10}$ Department of Pathology, Kanagawa Cancer Center Hospital, 1-1-2 Nakao, Asahi-ku, Yokohama 241-8515, Japan; ${ }^{11}$ Department of Pathology, Tokyo Metropolitan Geriatric Hospital and Institute of Gerontology, Tokyo, Japan; ${ }^{12}$ Cancer Science Institute of Singapore, Yong Loo Lin School of Medicine, National University of Singapore, 5 Lower Kent Ridge Road, Singapore 119074, Singapore; ${ }^{13}$ Genome Institute of Singapore, 60 Biopolis Street Genome \#02-01, Singapore 138672, Singapore and ${ }^{14}$ Cellular and Molecular Research, National Cancer Centre of Singapore, 11 Hospital Drive, Singapore 169610, Singapore
\end{abstract}

Background: Inhibitors of the epidermal growth factor (EGFR) signaling pathway have a major role in the treatment of KRAS wild-type colorectal cancer patients. The EGFR pathway has been shown to be activated in gastric cancer (GC). However, published data on KRAS and BRAF mutation status is limited in GC and has not been compared between GC from different geographic regions.

Methods: The prevalence of KRAS and BRAF mutations was established in 712 GC: 278 GC from the United Kingdom, 230 GC from Japan and 204 GC from Singapore. The relationship between KRAS/BRAF mutation status, DNA mismatch repair (MMR) status, clinicopathological variables and overall survival was analysed.

Results: Overall, 30 (4.2\%) GC carried a KRAS mutation. In total, 5.8\% of the UK GC, 4\% of Japan GC and 1.5\% of Singapore GC were KRAS mutant. KRAS mutant GC had fewer lymph node metastases in the UK cohort $(P=0.005)$ and were more frequent in elderly patients in the Japan cohort $(P=0.034)$. KRAS mutations were more frequent in MMR-deficient $G C$ in the UK and the Japanese cohort $(P<0.05)$. A BRAF mutation was only detected in a single Japanese GC.

Conclusions: This large multicentre study demonstrated that KRAS mutations and DNA MMR deficiency have a role in a small subgroup of GC irrespective of country of origin, suggesting that this subgroup of GC may have developed along a common pathway. Further studies need to establish whether concomitant mutations or amplifications of other EGFR signalling pathway genes may contribute to the activation of this pathway in GC.

*Correspondence: Dr T Yoshikawa; E-mail: yoshikawat@kcch.jp or Dr HI Grabsch; E-mail: h.i.grabsch@leeds.ac.uk

${ }^{15}$ These authors contributed equally to this paper.

Received 29 October 2012; revised 12 February 2013; accepted 19 February 2013; published online 19 March 2013

(c) 2013 Cancer Research UK. All rights reserved 0007-0920/13 
Despite a steady decline in incidence over the last decades, gastric cancer (GC) is still the fourth most common cancer worldwide and the second most common cause of cancerrelated death worldwide (Ferlay et al, 2010). Many GC patients present with locally advanced disease, which is treated with perioperative cytotoxic combination chemotherapy and surgery in the West (Cunningham et al, 2006) and surgery followed by chemotherapy in the East (Sakuramoto et al, 2007). However, even with multimodality treatment, the 5-year overall survival (OS) is less than $40 \%$ in advanced disease (Cunningham et al, 2006). Recent advances in targeted therapy demonstrated a survival benefit of trastuzumab in patients with HER2-positive inoperable GC (Bang et al, 2010). GC is characterised by geographical and molecular heterogeneity, which may potentially impact on the development of targeted therapy for this disease. Mutations of KRAS and BRAF, two major players of the epidermal growth factor (EGFR) signalling pathway, are known to have predictive value for therapies with antibodies targeting EGFR, such as panitumumab and cetuximab in patients with metastatic colorectal cancer (Misale et al, 2012). Recent studies demonstrated sensitivity to cetuximab in KRAS wild-type, EGFR-expressing GC cell lines and xenografts (Heindl et al, 2012; Hotz et al, 2012; Kneissl et al, 2012).

The first study reporting a KRAS mutation in GC was a case report in 1986 (Bos et al, 1986). Since then, 50 studies have investigated the KRAS mutation status in GC. More than $80 \%$ of studies were conducted in Asian GC patients and only seven of these studies included tumour material from more than 100 patients (Lee et al, 1995; Hao et al, 1998; Yoo et al, 2002; Lee et al, 2003; Yashiro et al, 2005; Tajima et al, 2006; Deng et al, 2012). The largest Western study to date included 82 GC patients (Brennetot et al, 2003), whereas the largest Asian study from Korea included 319 GC patients (Lee et al, 2003). All studies focussed on the mutation status of KRAS codons 12 and 13 using a number of different methods. The median KRAS mutation frequency of all GC cohorts was $6.5 \%$ (range: $0 \%-36 \%$ ) and was only slightly lower in the non-Asian GC (median 4\%, range 0\%-21\%) compared with Asian GC (median 6\%, range: 0\%-36\%).

Considering only studies with more than 100 GC patients, some of the authors reported a relationship between mutant KRAS and well-differentiated histology of GC (Yashiro et al, 2005), intestinal-type GC and higher pT stage (Yoo et al, 2002) and cancer location in the proximal third of the stomach (Lee et al, 1995).

Overall, the exact prevalence of KRAS mutations in locally advanced, resectable GC remains unknown and no definite conclusions can be drawn regarding the potential geographical heterogeneity or relationship of KRAS mutation status with clinicopathological data including survival. Furthermore, only a small number of studies investigated $B R A F$ mutation status in small GC patient cohorts and reported a frequency ranging from $0 \%$ to $11 \%$ with no relationship to histopathological variables (Kim et al, 2003; Lee et al, 2003; Oliveira et al, 2003; Wu et al, 2004; Sasao et al, 2006; Stella et al, 2009; Corso et al, 2011).

Results from three published studies, all investigating less than 100 GC patients, suggest that there might be an association between KRAS mutation status and DNA mismatch repair (MMR) status (Brennetot et al, 2003; Zhao et al, 2004; Gylling et al, 2007), although no causal relationship between DNA MMR status and KRAS mutation status has been shown to date (for review see Castagnola and Giaretti (2005)).

The aim of the current study was to establish the frequency of KRAS and BRAF mutations in GC in a large multicentre study, investigate the relationship between $K R A S / B R A F$ mutation status, DNA MMR status and clinicopathological variables including survival, and compare findings between GC from different geographic regions.

\section{MATERIALS AND METHODS}

Gastric cancer cohort from Leeds (UK). This study included 278 patients with sporadic gastric adenocarcinoma (GC) who underwent potentially curative surgery at the Department of Surgery, Leeds General Infirmary (Leeds, UK), between 1970 and 2004. None of the patients received any form of chemotherapy. Demographical, clinical and pathological data were retrieved from pathology reports, electronic patient hospital records and the Northern and Yorkshire Cancer Registry. Median follow-up time after surgery was 1.9 years, ranging from 0.11 to 20.48 years. Twenty-two patients died within 30 days after surgery and were excluded from survival analysis. Eight patients were lost from follow up. In total, 138 (49.6\%) patients died from GC during the study period. The study was approved by the Local Research Ethics Committee (LREC No. CA01/122).

Gastric cancer cohort from Yokohama (Japan). This study included 230 patients with stage II/III sporadic GC who underwent potentially curative surgery at Kanagawa Cancer Center Hospital (Yokohama, Japan) between 2001 and 2010. In total, 125 (54.3\%) patients received adjuvant chemotherapy (S-1 or Tegafur-uracil). Demographical, clinical and pathological data were retrieved from hospital records. Median follow-up time after surgery was 4.9 years, ranging from 0.5 to 10.4 years. None of the patients died within 30 days after surgery. Six patients were lost from follow up. Sixty-nine (30\%) patients died from GC during the study period. The study was approved by the Local Research Ethics Committee.

Gastric cancer cohort from Singapore (Singapore). This study included 204 Chinese patients with sporadic GC who underwent potentially curative surgery in Singapore (Singapore General Hospital, National University Hospital and Tan Tock Seng Hospital) between 1994 and 2008. Twenty-six (12.7\%) patients received adjuvant chemotherapy (5-Fluorouracil). Demographical, clinical and pathological data were retrieved from hospital records. Median follow-up time after surgery was 1.6 years, ranging from 0.2 to 13.1 years. None of the patients died within 30 days. Eight (3.9\%) patients were lost from follow up. In total, 106 (52\%) patients died from cancer and 11 patients died from other complications during the study period. This study was approved by the Local Research Ethics Committee and Institutional Review Board.

In all series, cases were staged according to TNM classification 7th edition (Sobin et al, 2009). Grade of differentiation was determined according to the WHO classification (WHO 2010) and morphological tumour type was classified according to Laurén's classification (Lauren, 1965).

DNA extraction. All haematoxylin/eosin-stained tissue sections from all resection specimens were reviewed by a histopathologist (HIG, NCTvG, YM, TArai, YK) and a representative formalinfixed, paraffin-embedded tissue block containing the highest density of primary adenocarcinoma was selected. The area of interest contained more than $30 \%$ tumour cells in all cases and was marked on the slide by the histopathologist to facilitate macrodissection. Depending on the size of the tumour up to five $10 \mu \mathrm{m}$ sections were cut, deparaffinised using a standard protocol and the marked area of interest was dissected using a sterile scalpel blade. Genomic DNA from the Yokohama and Leeds cases was extracted using a protocol based on the QIAmp DNA Micro Kit (Qiagen, Hilden, Germany) as described previously (Buffart et al, 2011) and using the DNeasy blood and tissue kit (Qiagen) for the Singapore cohort as described previously (Deng et al, 2012).

KRAS and BRAF mutation detection. In the Leeds GC cohort, mutation pre-screening using high-resolution melting technology followed by Sanger sequencing was used to detect KRAS codons 12, 
13, 61 and BRAF codon 600 mutations as described in detail previously (Kramer et al, 2009; Heideman et al, 2012). In the Yokohama GC cohort, pyrosequencing was used to determine the mutations status of KRAS codons 12, 13 and 61 as well as BRAF codon 600 as described previously (Richman et al, 2009). In the Singapore GC cohort, Sanger sequencing and MassARRAY technology (Sequenom Inc., San Diego, CA, USA) were used to determine the mutation status of KRAS codons 12 and 13 as described previously (Deng et al, 2012). KRAS codon 61 and BRAF mutation status were not assessed in the Singapore GC cohort.

DNA extracted from normal tissues from the same patient was genotyped for KRAS and/or BRAF mutation status from all cases with KRAS and/or BRAF mutation to distinguish between somatic and germline mutation.

\section{Assessment of the DNA MMR status}

Immunohistochemistry for MLH1, MSH2, PMS2 and MSH6. For the Singapore GC cohort, immunohistochemistry (IHC) was performed using the Leica BOND-MAX autostainer (Leica Microsystems Ltd, Milton Keynes, UK). Tissue sections were treated with Leica Bond epitope retrieval solution (ER-2, Leica, cat. no: AR9640) for 20 minutes (min) at $100{ }^{\circ} \mathrm{C}$ and incubated with primary antibodies, MLH1 (1:50, Cell, Marque, Rocklin, CA, USA, cat. no: 285M-16), MSH2 (1:50, Biocare Medical, Concord, CA, USA, cat. no: CM219), MSH6 (1:150, Biocare Medical, cat.no: CM265) and PMS2 (1:150, Leica, cat. no: NCL-PMS2) for $20 \mathrm{~min}$ at room temperature. Leica Bond polymer refine DAB detection system was used according to the instructions of the manufacturer. Sections were counterstained with haematoxylin, dehydrated and mounted.

For the Yokohama GC cohort, IHC was performed manually as described previously (Grabsch et al, 2010) using $0.1 \mathrm{M}$ citrate buffer $\mathrm{pH} 6.0$ for antigen retrieval in a microwavable pressure cooker. Slides were incubated with primary antibodies, MLH1 (1:50, overnight at $4{ }^{\circ} \mathrm{C}$, BD Pharmingen, Oxford, UK, cat. no: 550838), MSH2 (1:70, $60 \mathrm{~min}$ at $37^{\circ} \mathrm{C}$, Calbiochem, Watford, UK, cat. no: NA27), MSH6 (1:50, overnight at $4{ }^{\circ} \mathrm{C}$, Invitrogen, Paisley, UK, cat. no: $18-0443)$ and PMS2 $\left(1: 25\right.$, overnight at $4^{\circ} \mathrm{C}, \mathrm{BD}$ Pharmingen cat. no: 556415). The Dako Real streptavidin-biotin detection kit (Dako, Ely, UK) or a tyramine-based amplification system and DAB were used as described previously (Grabsch et al, 2010). Sections were counterstained with haematoxylin, dehydrated and mounted.

The scoring system used was the same for both cohorts. GC with positive stained tumour cell nuclei were classified as MMRproficient. GC were only classified as 'negative' (MMR-deficient) if the tissue section contained an internal positive control such as lymphocytes.

Microsatellite analysis. The MSI Multiplex System Version 1.2 (Promega, Southampton, UK, cat. no MD1641) was used for the detection of microsatellite instability according to the instructions of the manufacturer. This kit allows the co-amplification of BAT25, BAT-26, NR-21, NR24 and MONO-27 from the same input DNA sample. The PCR products were separated by capillary electrophoresis using an ABI PRISM 3100 DNA sequencer and analysed with GeneMapper 3.5 software (Applied Biosystems, Paisley, UK). As the overall frequency of microsatellite instability was very low in the current cohorts, no distinction was made between low and high microsatellite instability. The kit includes a genomic DNA sample, which served as positive control, and nuclease-free water, which was used as negative control.

In 112 GC patients from Singapore, the MMR status was determined by IHC as well as by microsatellite analysis. All Singapore GC cases, which were negative for at least one of the MMR proteins by IHC, showed microsatellite instability and all cases positive for all four MMR proteins by IHC were microsatellite stable, a finding that is consistent with the published literature. A decision was therefore made to perform IHC on the Yokohama GC patients and microsatellite analysis on the Leeds GC patients, as available material was limited.

A case was classified as 'MMR-deficient' if either one of the MMR proteins was negative by IHC or the case showed microsatellite instability.

Statistical analysis. Statistical analyses were performed using the Statistical Package for the Social Sciences (SPSS 15.0 for Windows, Chicago, USA).

Comparisons between the mutation status, the DNA MMR status and the clinicopathological variables were performed using the Mann-Whitney $U$-test (for two groups) or the Kruskal-Wallis test (for more than two groups). Analyses of overall survival (OS) were performed using the Kaplan-Meier method and differences between groups were tested by the log-rank test. Data from patients who died within 30 days after surgery were excluded from survival analysis. $P$-values less than 0.05 were considered significant.

\section{RESULTS}

KRAS and BRAF mutation status in the Leeds GC cohort. KRAS exon 1 (codons 12 and 13) mutation data were available from 276 GC patients. Two (0.7\%) GC failed to amplify. KRAS exon 2 (codon 61) data were available from 270 (97\%) of the tested 278 GC. BRAF codon 600 data were available from 264 (95\%) of the tested 278 GC.

A KRAS mutation was found in 16 (5.8\%) GC. Twelve (75\%) mutations occurred in KRAS codon 12, 2 (13\%) in KRAS codon 13 and $1(12 \%)$ in KRAS codon 61 . The most common mutation was p.G12D, which was found in five (30\%) GC, followed by p.G12V (4 GC) and p.G12A (2 GC). KRAS mutations p.G12C, p.G13C, p.G13D and p.Q61H were found in one GC each. No concurrent KRAS mutations were seen. None of the Leeds GC had a BRAF V600E mutation. With the exception of a KRAS p.V8V polymorphism (rs147406419), which was found in the tumour and normal DNA from one patient, all matched normal DNA showed KRAS and BRAF wild-type.

KRAS and BRAF mutation status in the Yokohama GC cohort. KRAS exon 1 (codons 12 and 13) and exon 2 (codon 61) mutation data were available from all $230 \mathrm{GC}$ patients. $B R A F$ codon 600 data were available from 227 (99\%) of the tested 230 GC.

A KRAS mutation was found in 10 (4\%) GC. Six (60\%) mutations were located in KRAS codon 12 and four (40\%) in KRAS codon 13. No mutation was found in KRAS codon 61. The most common mutation was p.G12D, which was found in six (60\%) GC, the remaining four GC had a p.G13D mutation. No concurrent KRAS mutations were seen. One (0.4\%) GC had a BRAF V600E mutation and was KRAS wild-type at the same time. All matched normal DNA showed KRAS and BRAF wild-type.

KRAS and BRAF mutation status in the Singapore GC cohort. KRAS exon 1 (codons 12 and 13) mutation data were available from 204 GC patients. Three (1.5\%) GC showed a KRAS mutation. Two mutations were located in codon 12 (p.G12C and p.G12D) and one in codon 13 (p.G13D). All matched normal DNA showed KRAS wild-type.

Comparison of the KRAS/BRAF mutation status between the cohorts. There was no statistically significant difference in the overall frequency of KRAS mutations between the three GC cohorts with 5.8\% (Leeds), 4\% (Yokohama) and 1.5\% (Singapore).

A total of $75 \%$ of KRAS mutations were located in codon 12 in the Leeds cohort compared with $60 \%$ in the Yokohama cohort and $67 \%$ in the Singapore cohort. A KRAS codon 61 mutation was only 
found in the Leeds cohort, whereas a BRAF mutation was only found in the Yokohama cohort. No concurrent mutations were found in any of the patients.

KRAS/BRAF mutation status and clinicopathological characteristics. Owing to the small number of mutations found, GC were categorised as 'KRAS wild-type' or 'KRAS mutant' for statistical analyses. When results from all three GC cohorts were combined for analyses, no significant relationship was found between KRAS mutation status and clinicopathological variables.

Because there were only three KRAS mutant GC in the Singapore cohort, no statistical analyses were performed within this cohort. One of the KRAS mutant Singapore GC was from a male patient and was staged as pT3N0M0. The two other KRAS mutant Singapore GCs were from female patients and both staged as pT4N1M0. All KRAS mutations in the Singapore GC cohort occurred in moderately differentiated intestinal-type GC and in patients younger than 70 years.

In the Leeds GC cohort, the only significant relationship found was that KRAS mutations were more common in Leeds GC with lower lymph node category ( $\mathrm{pN}, P=0.005$, see Table 1 ). None of the patients with more than six lymph node metastases $(\mathrm{pN} 3 \mathrm{a} / \mathrm{b})$ had KRAS mutations. A total of $81 \%$ of the KRAS mutant Leeds GC were of intestinal-type histology and $88 \%$ were locally advanced cancers with infiltration of the subserosa or beyond. However, due to the overall small number of cancers with KRAS mutations, these findings were not statistically significant. In contrast to the Singapore GC, $69 \%$ of KRAS mutant Leeds GC occurred in patients older than 70 years at the time of diagnosis.

In the Yokohama GC cohort, KRAS mutations were more frequent in the elderly patients aged $\geqslant 70$ years $(P=0.034)$. There was a trend for a higher KRAS mutation frequency in welldifferentiated Yokohama GC $(P=0.063)$. The single $B R A F$-mutant Yokohama GC occurred in a 61-year-old male patient, was of poorly differentiated type histology and staged as pT3N2M0.

There was no relationship with any of the other clinicopathological variables tested (see Table 1).

KRAS mutation status and overall survival. As expected, depth of tumour invasion ( $\mathrm{T}$ category) and lymph node status ( $\mathrm{N}$ category) were significant independent predictors of prognosis in all GC cohorts (data not shown).

Univariate overall survival (OS) analysis showed no significant difference when patients were stratified by KRAS mutation status irrespective of whether the results from all cohorts were combined for analysis or cohorts were analysed individually.

In the Leeds cohort, the OS rate at 3 and 5 years after surgery in patients with KRAS mutant GC was $42.9 \%$ and $35.7 \%$, respectively, compared with $37.9 \%$ and $31.2 \%$ in patients with KRAS wild-type GC, $P=0.5057$. In the Yokohama cohort, the OS rate at 3 and 5 years after surgery in patients with KRAS mutant GC was $81.8 \%$ and $71.6 \%$, respectively, compared with $74.1 \%$ and $59.5 \%$ in patients with KRAS wild-type GC, $P=0.5850$. There was also no significant difference in survival between patients with or without KRAS mutant GC in the Yokohama cohort when survival was analysed separately in patients treated with or without adjuvant chemotherapy. In the Singapore cohort, the OS rate at 3 and 5 years after surgery in patients with KRAS mutant GC was $66.7 \%$ for both time points compared with $51.7 \%$ and $47.3 \%$ in patients with KRAS wild-type GC.

KRAS/BRAF mutation status and DNA MMR status. MMR status data were available from 264 Leeds GC of which 25 (9\%) were classified as MMR-deficient. A higher incidence of KRAS mutations were noted in the MMR-deficient GC: 11 (5\%) of the MMR-proficient and 5 (20\%) of the MMR-deficient Leeds GC had a KRAS mutation $(P=0.002$, Table 1$)$. Four of the five KRAS
mutant/MMR-deficient GC were intestinal-type GC, one showed a mixed histology.

MMR status data were available from 230 Yokohama GC of which 21 (9\%) were classified as MMR-deficient. A higher incidence of KRAS mutations were noted in the MMR-deficient GC: $11(3 \%)$ of the MMR-proficient and 3 (14\%) of the MMRdeficient Yokohama GC had a KRAS mutation $(P=0.019$, Table 1). One of the KRAS mutant/MMR-deficient GC was an intestinal-type GC, one a diffuse-type GC and one a mucinous GC. The Yokohama GC with BRAF mutation, which was the only case with BRAF mutation in the whole series, was classified as MMRproficient as all four IHC markers were positive.

MMR status data were available from 122 Singapore GC of which $17(14 \%)$ were classified as MMR-deficient. Of the three KRAS mutant GC, one showed MMR deficiency, one was classified as MMR-proficient and no data were available from the third case.

\section{DISCUSSION}

Five-year survival of patients with locally advanced GC is still poor in the East and the West even after modern multimodality treatment combining radical surgical resection with cytotoxic chemotherapy (Cunningham et al, 2006; Sakuramoto et al, 2007). Several clinical studies are underway to evaluate the potential efficacy of EGFR inhibitors in patients with metastatic oesophagogastric cancer, none of them is currently using a biomarker to select patients (Okines et al, 2011). In colorectal cancer, benefit from EGFR inhibitors has been restricted to patients with KRAS wild-type cancer (Misale et al, 2012). The determination of the prevalence of KRAS/BRAF mutation in a sufficiently large series of GC from different geographic regions appears to be an essential prerequisite for further worldwide clinical development of EGFRdirected therapy in GC.

The current study is the largest study to date investigating KRAS and $B R A F$ mutation status and DNA MMR status in patients with locally advanced resectable GC originating from three different countries with different GC incidence, Caucasian patients from the UK, Japanese patients and Chinese patients from Singapore. A $B R A F$ mutation was found in a single GC from Yokohama confirming the absence or very low frequency of $B R A F$ mutations in GC reported previously (Lee et al, 2003; Oliveira et al, 2003; Zhao et al, 2004).

The prevalence of KRAS mutation in all primary resectable GC of this study was $4 \%$ and statistically not different between the different GC cohorts. From this result, which is in concordance with the published GC literature on KRAS mutation frequency (Hongyo et al, 1995; Lee et al, 1995; Zhao et al, 2004), there is no evidence to suggest that KRAS mutation frequency is related to GC incidence, aetiology or ethnicity, factors which are all significantly different in countries from the East and the West (Ferlay, 2010). Furthermore, in all investigated cohorts, KRAS mutation frequency was statistically not related to gender, tumour location, depth of invasion, grade of differentiation or tumour morphology. However, looking at the subgroup of all KRAS mutant GC investigated in the current study, almost two-third of KRAS mutant GC were intestinal-type GC, which is consistent with other studies (Miki et al, 1991; Yoo et al, 2002; Corso et al, 2011). It is difficult to compare our findings to the current GC literature as the studies published so far are contradictory. As such, KRAS mutations in GC were described as being exclusively seen in males (Liu et al, 2009) but also to be more common in females (Corso et al, 2011), more frequent in well-differentiated GC (Kihana et al, 1991; Hiyama et al, 2002; Yashiro et al, 2005), in distal cancers (Zhao et al, 2004), in proximal cancers (Lee et al, 1995), in early-stage cancers (Hongyo et al, 1995; Liu et al, 2009), whereas other studies found 


\begin{tabular}{|c|c|c|c|c|c|c|c|c|c|c|c|c|c|c|}
\hline & \multicolumn{6}{|c|}{ Leeds gastric cancer } & & \multicolumn{6}{|c|}{ Yokohama gastric cancer } & \\
\hline & \multicolumn{2}{|c|}{ Total } & \multicolumn{2}{|c|}{ KRAS wild-type } & \multicolumn{2}{|c|}{ KRAS mutated } & & \multicolumn{2}{|c|}{ Total } & \multicolumn{2}{|c|}{ KRAS wild-type } & \multicolumn{2}{|c|}{ KRAS mutated } & \\
\hline & $n$ & $\%$ & $n$ & $\%$ & $n$ & $\%$ & $\boldsymbol{P}$-value & $n$ & $\%$ & $n$ & $\%$ & $n$ & $\%$ & $P$-value \\
\hline \multicolumn{15}{|c|}{ Age group } \\
\hline$<70$ years & 112 & 41 & 107 & 96 & 5 & 4 & 0.434 & 161 & 70 & 157 & 97 & 4 & 3 & 0.034 \\
\hline$\geqslant 70$ years & 164 & 59 & 153 & 93 & 11 & 7 & & 69 & 30 & 63 & 91 & 6 & 9 & \\
\hline \multicolumn{15}{|l|}{ Gender } \\
\hline Male & 164 & 59 & 155 & 95 & 9 & 5 & 0.791 & 162 & 70 & 155 & 96 & 7 & 4 & 0.975 \\
\hline Female & 112 & 41 & 105 & 94 & 7 & 6 & & 68 & 30 & 65 & 96 & 3 & 4 & \\
\hline \multicolumn{15}{|c|}{ Tumour location } \\
\hline Proximal & 66 & 24 & 64 & 97 & 2 & 3 & 0.421 & 69 & 30 & 67 & 97 & 2 & 3 & 0.693 \\
\hline Mid & 72 & 26 & 66 & 92 & 6 & 8 & & 93 & 40 & 89 & 96 & 4 & 4 & \\
\hline Distal & 122 & 45 & 115 & 94 & 7 & 6 & & 68 & 30 & 64 & 94 & 4 & 6 & \\
\hline Stump & 8 & 3 & 7 & 88 & 1 & 12 & & 0 & 0 & 0 & 0 & 0 & 0 & \\
\hline L. plastica & 5 & 2 & 5 & 100 & 0 & 0 & & 0 & 0 & 0 & 0 & 0 & 0 & \\
\hline \multicolumn{15}{|c|}{ Depth of invasion (pT) } \\
\hline $\mathrm{pT1a/b}$ & 20 & 7 & 18 & 90 & 2 & 10 & 0.735 & 8 & 4 & 8 & 100 & 0 & 0 & 0.214 \\
\hline pT2 & 23 & 8 & 23 & 100 & 0 & 0 & & 42 & 18 & 38 & 91 & 4 & 9 & \\
\hline pT3 & 80 & 29 & 74 & 93 & 6 & 7 & & 30 & 13 & 30 & 100 & 0 & 0 & \\
\hline pT4a/b & 153 & 55 & 145 & 95 & 8 & 5 & & 150 & 65 & 144 & 96 & 6 & 4 & \\
\hline \multicolumn{15}{|c|}{ Lymph node status (pN) } \\
\hline pNO & 85 & 31 & 76 & 89 & 9 & 11 & 0.005 & 40 & 17 & 40 & 100 & 0 & 0 & 0.160 \\
\hline pN1 & 51 & 19 & 48 & 94 & 3 & 6 & & 55 & 24 & 51 & 93 & 4 & 7 & \\
\hline pN2 & 54 & 20 & 50 & 93 & 4 & 7 & & 62 & 27 & 61 & 98 & 1 & 2 & \\
\hline $\mathrm{pN3a/b}$ & 84 & 31 & 84 & 100 & 0 & 0 & & 73 & 32 & 68 & 93 & 5 & 7 & \\
\hline \multicolumn{15}{|c|}{ Grade of differentiation } \\
\hline G1 & 31 & 11 & 28 & 90 & 3 & 10 & 0.768 & 23 & 10 & 20 & 87 & 3 & 13 & 0.063 \\
\hline G2 & 88 & 32 & 84 & 96 & 4 & 4 & & 53 & 23 & 50 & 94 & 3 & 6 & \\
\hline G3 & 156 & 57 & 147 & 94 & 9 & 6 & & 154 & 67 & 150 & 97 & 4 & 3 & \\
\hline \multicolumn{15}{|c|}{ Laurén classification } \\
\hline Intestinal & 178 & 65 & 165 & 93 & 13 & 7 & 0.150 & 120 & 52 & 117 & 97 & 3 & 3 & 0.151 \\
\hline Diffuse & 60 & 22 & 58 & 97 & 2 & 3 & & 110 & 48 & 103 & 94 & 7 & 6 & \\
\hline Mixed & 38 & 14 & 37 & 97 & 1 & 3 & & 0 & 0 & 0 & 0 & 0 & 0 & \\
\hline \multicolumn{15}{|c|}{ Mismatch repair status } \\
\hline Proficient & 239 & 91 & 228 & 96 & 11 & 4 & 0.002 & 209 & 91 & 202 & 97 & 7 & 3 & 0.019 \\
\hline Deficient & 25 & 9 & 20 & 80 & 5 & 20 & & 21 & 9 & 18 & 86 & 3 & 14 & \\
\hline
\end{tabular}

no such associations (Nanus et al, 1990; Arber et al, 2000; Lee et al, 2003). All previous studies suffer from investigating a relatively small number of GC patients making the interpretation of any statistical analysis difficult.

The higher frequency of KRAS mutations in patients with lower $\mathrm{pN}$ category in the Leeds GC cohort confirms a previous report from a small cohort of Chinese GC (Liu et al, 2009). It is currently unclear why no such relationship was seen in the Japanese GC cohort. KRAS mutation status was not related with survival in any of the three GC cohorts confirming a previous report in 140 Japanese GCs (Lee et al, 1995).

The current study showed that KRAS mutations are more frequent but not exclusive to MMR-deficient GC confirming results from a small previous study (Zhao et al, 2004). Other previous studies did not identify KRAS mutations in MMR-proficient GC, which could be related to the very small number of GC investigated (Brennetot et al, 2003; Gylling et al, 2007). Interestingly, these findings in GC are in contrast to results from studies in colorectal cancer where a lower incidence of KRAS mutations in MMR-deficient cancers has been described (Hutchins et al, 2011). Although this is currently the largest series of GC investigating more than $700 \mathrm{GC}$ for MMR status and KRAS mutation status, the total number of GC showing KRAS mutation or MMR deficiency and KRAS mutation is still very small making interpretation difficult. However, the existence of a small subgroup of GC with distinct molecular characteristics may be related to the known heterogeneity of GC.

Further studies are required to characterise the KRAS mutant GC subgroup at a molecular level in order to better understand the biological effects of KRAS mutation in GC. It would be of particular interest to establish whether the RTK/RAS signalling pathway might be activated in GC due to multiple concomitant 
mutations of genes related to RTK/RAS signalling or concomitant gene amplifications also present in only small subsets of GC (Deng et $a l, 2012)$. The presence of up to $40 \%$ concomitant EGFR pathway-related mutations has been reported in a small study $(n=63)$ of GC very recently (Corso et al, 2011). However, BRAF mutations do not seem to have any role in GC.

The current study has some limitations that are mainly related to the fact that this was a retrospective study. Although this is a very large series of GC with more than 700 patients, the interpretation of the results remains challenging, as the prevalence of KRAS mutation, DNA MMR deficiency and combined KRAS mutation/DNA MMR deficiency is relatively low. Hence, even this large multicentre study may still be underpowered to detect an association between KRAS mutation and overall survival. However, there was a trend in the current study that the presence of a KRAS mutation was associated with better overall survival in GC patients, which is in contrast to studies in colorectal cancer.

For the current study, we used DNA from a single tissue block found to be representative of the primary cancer based on morphology. Gastric cancer is known to be very heterogeneous and thus, by using only one block we may have underestimated the true mutation frequency. However, there is currently no evidence in the literature to support that $K R A S / B R A F$ mutations are heterogeneous in GC or that the frequency differs between primary cancer and lymph node metastasis. For practical reasons, we have used different methods to evaluate the KRAS mutation status in the different patient cohorts. However, all methods have shown to be able to detect mutations in samples with less than $10 \%$ mutated tumour cells (Heideman et al, 2012) and all samples used for extraction had in effect more than $30 \%$ of tumour cells. Unfortunately, we do not have access to material from clinical studies investigating the efficacy of EGFR inhibitors. Hence, it remains to be shown whether KRAS mutation status predicts treatment response in GC patients.

In summary, this is the largest study to date investigating the KRAS and BRAF mutation status as well as DNA MMR status in locally advanced, resectable GC from the East and the West. The study confirms that KRAS mutations and DNA MMR deficiency have a role in a small subgroup of GC irrespective of country of origin of the patient.

These data suggest that neither KRAS mutations nor DNA MMR deficiency are related to the very different GC incidence in the East and the West. Similar KRAS mutation frequency and similar incidence of DNA MMR deficiency in GC patients from multiple cohorts may suggest that these particular subgroup of GC may have develop along a common yet to be identified pathway. Further molecular characterisation of these GC subgroups is needed to understand the biological effect of KRAS mutations and DNA MMR in GC.

\section{ACKNOWLEDGEMENTS}

We thank Marije Doeleman and Marinda van Moorsel for expert technical assistance. This work was supported by grants from the Sasakawa Foundation UK, Pathological Society of Great Britain and Ireland, Cancer Research UK grant C37059/A11941, NonProfit Organizations Kanagawa Standard Anti-cancer Therapy Support System (Yokohama, Japan) and the Health Insurance Company (LPT grant, The Netherlands).

\section{REFERENCES}

Arber N, Shapira I, Ratan J, Stern B, Hibshoosh H, Moshkowitz M, Gammon M, Fabian I, Halpern Z (2000) Activation of c-K-ras mutations in human gastrointestinal tumors. Gastroenterology 118: 1045-1050.
Bang YJ, Van Cutsem E, Feyereislova A, Chung HC, Shen L, Sawaki A, Lordick F, Ohtsu A, Omuro Y, Satoh T, Aprile G, Kulikov E, Hill J, Lehle M, Ruschoff J, Kang YK (2010) Trastuzumab in combination with chemotherapy versus chemotherapy alone for treatment of HER2-positive advanced gastric or gastro-oesophageal junction cancer (ToGA): a phase 3, open-label, randomised controlled trial. Lancet 376: 687-697.

Bos JL, MV-d Vries, Marshall CJ, Veeneman GH, van Boom JH, van der Eb AJ (1986) A human gastric carcinoma contains a single mutated and an amplified normal allele of the Ki-ras oncogene. Nucleic Acids Res 14: 1209-1217.

Brennetot C, Duval A, Hamelin R, Pinto M, Oliveira C, Seruca R, Schwartz S (2003) Frequent ki-ras mutations in gastric tumors of the MSI phenotype. Gastroenterology 125: 1282-1283.

Buffart TE, Louw M, van Grieken NC, Tijssen M, Carvalho B, Ylstra B, Grabsch H, Mulder CJ, van de Velde CJ, van der Merwe SW, Meijer GA (2011) Gastric cancers of Western European and African patients show different patterns of genomic instability. BMC Med Genomics 4: 7.

Castagnola P, Giaretti W (2005) Mutant KRAS, chromosomal instability and prognosis in colorectal cancer. Biochim Biophys Acta Rev Cancer 1756: $115-125$.

Corso G, Velho S, Paredes J, Pedrazzani C, Martins D, Milanezi F, Pascale V, Vindigni C, Pinheiro H, Leite M, Marrelli D, Sousa S, Carneiro F, Oliveira C, Roviello F, Seruca R (2011) Oncogenic mutations in gastric cancer with microsatellite instability. Eur J Cancer 47: 443-451.

Cunningham D, Allum WH, Stenning SP, Thompson JN, Van de Velde CJ, Nicolson M, Scarffe JH, Lofts FJ, Falk SJ, Iveson TJ, Smith DB, Langley RE, Verma M, Weeden S, Chua YJ, Participants MT (2006) Perioperative chemotherapy versus surgery alone for resectable gastroesophageal cancer. $N$ Engl J Med 355: 11-20.

Deng N, Goh LK, Wang H, Das K, Tao J, Tan IB, Zhang S, Lee M, Wu J, Lim KH, Lei Z, Goh G, Lim QY, Tan AL, Sin Poh DY, Riahi S, Bell S, Shi MM, Linnartz R, Zhu F, Yeoh KG, Toh HC, Yong WP, Cheong HC, Rha SY, Boussioutas A, Grabsch H, Rozen S, Tan P (2012) A comprehensive survey of genomic alterations in gastric cancer reveals systematic patterns of molecular exclusivity and co-occurrence among distinct therapeutic targets. Gut 61: 673-684.

Ferlay J, Shin HR, Bray F, Forman D, Mathers C, Parkin DM (2010) GLOBOCAN 2008 v2.0, Cancer Incidence and Mortality Worldwide: IARC CancerBase No. 10 [Internet]. Lyon, France:International agency for Research on Cancer; 2010. Available from http://globocan.iarc.fr, accessed on 15/October/2012.

Grabsch H, Sivakumar S, Gray S, Gabbert HE, Müller W (2010) HER2 expression in gastric cancer: Rare, heterogeneous and of no prognostic value conclusions from 924 cases of two independent series. Cell Oncol 32: 57-65.

Gylling A, Abdel-Rahman WM, Juhola M, Nuorva K, Hautala E, Järvinen HJ, Mecklin J-P, Aarnio M, Peltomäki P (2007) Is gastric cancer part of the tumour spectrum of hereditary non-polyposis colorectal cancer? A molecular genetic study. Gut 56: 926-933.

Hao Y, Zhang J, Lu Y, Yi C, Qian W, Cui J (1998) The role of ras gene mutation in gastric cancer and precancerous lesions. J Tongji Med Univ 18: $141-144$.

Heideman DAM, Lurkin I, Doeleman M, Smit EF, Verheul HM, Meijer GA, Snijders PJ, Thunnissen E, Zwarthoff EC (2012) KRAS and BRAF mutation analysis in routine molecular diagnostics: comparison of three testing methods on formalin-fixed, paraffin-embedded tumor-derived DNA. J Mol Diagn 14: 247-255.

Heindl S, Eggenstein E, Keller S, Kneissl J, Keller G, Mutze K, Rauser S, Gasteiger G, Drexler I, Hapfelmeier A, Hofler H, Luber B (2012) Relevance of MET activation and genetic alterations of KRAS and E-cadherin for cetuximab sensitivity of gastric cancer cell lines. J Cancer Res Clin Oncol 138: $843-858$.

Hiyama T, Haruma K, Kitadai Y, Masuda H, Miyamoto M, Tanaka S, Yoshihara M, Shimamoto F, Chayama K (2002) K-ras mutation in Helicobacter pylori-associated chronic gastritis in patients with and without gastric cancer. Int J Cancer 97: 562-566.

Hongyo T, Buzard GS, Palli D, Weghorst CM, Amorosi A, Galli M, Caporaso NE, Fraumeni JF, Rice JM (1995) Mutations of the K-ras and p53 genes in gastric adenocarcinomas from a high-incidence region around Florence, Italy. Cancer Res 55: 2665-2672.

Hotz B, Keilholz U, Fusi A, Buhr HJ, Hotz HG (2012) In vitro and in vivo antitumor activity of cetuximab in human gastric cancer cell lines in relation to epidermal growth factor receptor (EGFR) expression and mutational phenotype. Gastric Cancer 15: 252-264. 
Hutchins G, Southward K, Handley K, Magill L, Beaumont C, Stahlschmidt J, Richman S, Chambers P, Seymour M, Kerr D, Gray R, Quirke P (2011) Value of mismatch repair, KRAS, and BRAF mutations in predicting recurrence and benefits from chemotherapy in colorectal cancer. J Clin Oncol 29: 1261-1270.

Sobin LH, Gospodarowicz MK, Witterkind CH (eds), International Union Against Cancer (UICC) TNM Classification of Malignant Tumors, 7th ed. Wiley-Blackwell: Oxford, United Kingdom, 2009.

Kihana T, Tsuda H, Hirota T, Shimosato Y, Sakamoto H, Terada M, Hirohashi S (1991) Point mutation of c-Ki-ras oncogene in gastric adenoma and adenocarcinoma with tubular differentiation. Jpn J Cancer Res 82: 308-314.

Kim I-J, Park J-H, Kang H, Shin Y, Park H-W, Park H-R, Ku J-L, Lim S-B, Park J-G (2003) Mutational analysis of BRAF and K-ras in gastric cancers: absence of BRAF mutations in gastric cancers. Hum Genet 114: 118-120.

Kneissl J, Keller S, Lorber T, Heindl S, Keller G, Drexler I, Hapfelmeier A, Hofler H, Luber B (2012) Association of amphiregulin with the cetuximab sensitivity of gastric cancer cell lines. Int J Oncol 41: 733-744.

Kramer D, Thunnissen FB, Gallegos-Ruiz MI, Smit EF, Postmus PE, Meijer CJ, Snijders PJ, Heideman DA (2009) A fast, sensitive and accurate high resolution melting (HRM) technology-based assay to screen for common K-ras mutations. Cell Oncol 31: 161-167.

Lauren P (1965) The two histological main types of gastric carcinoma: diffuse and so called intestinal-type carcinoma. Acta Pathol Microbiol Scand 64: $31-49$.

Lee KH, Lee JS, Suh C, Kim SW, Kim SB, Lee JH, Lee MS, Park MY, Sun HS, Kim SH (1995) Clinicopathologic significance of the K-ras gene codon 12 point mutation in stomach cancer. An analysis of 140 cases. Cancer 75 : 2794-2801.

Lee SH, Lee JW, Soung YH, Kim HS, Park WS, Kim SY, Lee JH, Park JY, Cho YG, Kim CJ, Nam SW, Kim SH, Lee JY, Yoo NJ (2003) BRAF and KRAS mutations in stomach cancer. Oncogene 22: 6942-6945.

Liu ZM, Liu LN, Li M, Zhang QP, Cheng SH, Lu S (2009) Mutation detection of KRAS by high-resolution melting analysis in Chinese with gastric cancer. Oncol Rep 22: 515-520.

Miki H, Ohmori M, Perantoni AO, Enomoto T (1991) K-ras activation in gastric epithelial tumors in Japanese. Cancer Lett 58: 107-113.

Misale S, Yaeger R, Hobor S, Scala E, Janakiraman M, Liska D, Valtorta E, Schiavo R, Buscarino M, Siravegna G, Bencardino K, Cercek A, Chen CT, Veronese S, Zanon C, Sartore-Bianchi A, Gambacorta M, Gallicchio M, Vakiani E, Boscaro V, Medico E, Weiser M, Siena S, Di Nicolantonio F, Solit D, Bardelli A (2012) Emergence of KRAS mutations and acquired resistance to anti-EGFR therapy in colorectal cancer. Nature 486: $532-536$.

Nanus DM, Kelsen DP, Mentle IR, Altorki N, Albino AP (1990) Infrequent point mutations of ras oncogenes in gastric cancers. Gastroenterology $\mathbf{9 8}$ : 955-960.
Okines A, Cunningham D, Chau I (2011) Targeting the human EGFR family in esophagogastric cancer. Nat Rev Clin Oncol 8: 492-503.

Oliveira C, Pinto M, Duval A, Brennetot C, Domingo E, Espin E, Armengol M, Yamamoto H, Hamelin R, Seruca R, Schwartz Jr S (2003) BRAF mutations characterize colon but not gastric cancer with mismatch repair deficiency. Oncogene 22: 9192-9196.

Richman SD, Seymour MT, Chambers P, Elliott F, Daly CL, Meade AM, Taylor G, Barrett JH, Quirke P (2009) KRAS and BRAF mutations in advanced colorectal cancer are associated with poor prognosis but do not preclude benefit from oxaliplatin or irinotecan: results from the MRC FOCUS trial. J Clin Oncol 27: 5931-5937.

Sakuramoto S, Sasako M, Yamaguchi T, Kinoshita T, Fujii M, Nashimoto A, Furukawa H, Nakajima T, Ohashi Y, Imamura H, Higashino M, Yamamura Y, Kurita A, Arai K (2007) Adjuvant chemotherapy for gastric cancer with S-1, an oral fluoropyrimidine. N Engl J Med 357: 1810-1820.

Sasao S, Hiyama T, Tanaka S, Yoshihara M, Yasui W, Chayama K (2006) Clinicopathologic and genetic characteristics of gastric cancer in young male and female patients. Oncol Rep 16: 11-15.

Stella G, Rojas Llimpe F, Barone C, Falcone A, Di Fabio F, Martoni A, Lamba S, Ceccarelli C, Siena S, Bardelli A, Pinto C (2009) KRAS and BRAF mutational status as response biomarkers to cetuximab combination therapy in advanced gastric cancer patients. ASCO Meet Abstr 27(15S): e15503.

Tajima Y, Yamazaki K, Makino R, Nishino N, Aoki S, Kato M, Morohara K, Kaetsu T, Kusano M (2006) Gastric and intestinal phenotypic marker expression in early differentiated-type tumors of the stomach: clinicopathologic significance and genetic background. Clin Cancer Res 12: 6469-6479.

WHO (2010) WHO classification of tumours of the digestive system. 4 edn. IARC: Lyon.

Wu M, Semba S, Oue N, Ikehara N, Yasui W, Yokozaki H (2004) BRAF/K-ras mutation, microsatellite instability, and promoter hypermethylation of hMLH1/MGMT in human gastric carcinomas. Gastric Cancer 7: 246-253.

Yashiro M, Nishioka N, Hirakawa K (2005) K-ras mutation influences macroscopic features of gastric carcinoma. J Surg Res 124: 74-78.

Yoo J, Park SY, Robinson RA, Kang SJ, Ahn WS, Kang CS (2002) RAS gene mutations and expression of RAS signal transduction mediators in gastric adenocarcinomas. Arch Pathol Lab Med 126: 1096-1100.

Zhao W, Chan TL, Chu KM, Chan AS, Stratton MR, Yuen ST, Leung SY (2004) Mutations of BRAF and KRAS in gastric cancer and their association with microsatellite instability. Int J Cancer 108: 167-169.

This work is published under the standard license to publish agreement. After 12 months the work will become freely available and the license terms will switch to a Creative Commons AttributionNonCommercial-Share Alike 3.0 Unported License. 